\title{
Characterizing variants of qualitative Sugeno integrals in a totally ordered Heyting algebra
}

\author{
Didier Dubois $^{1}$ Agnès Rico $^{2}$ Bruno Teheux $^{3}$ Henri Prade ${ }^{1}$ \\ ${ }^{1}$ IRIT, Université Paul Sabatier 31062 Toulouse cedex 9 (France) \\ ${ }^{2}$ ERIC, Université Claude Bernard Lyon 169100 Villeurbanne (France) \\ ${ }^{3}$ Mathematics Research Unit, FSTC, University of Luxembourg, L-1359 Luxembourg (Luxembourg)
}

\begin{abstract}
Sugeno integral is one of the basic aggregation operations on a qualitative scale where only minimum and maximum, as well as order-reversing maps are allowed. Recently some variants of this aggregation operation, named soft and drastic integrals, have been introduced in a previous work by three of the authors. In these operations, importance weights play the role of tolerance thresholds enabling full satisfaction if ratings pass them. These new aggregation operations use residuated implications, hence need a slightly richer structure, and are part of larger family of qualitative aggregations. Based on some properties laid bare in a previous work, this paper proposes characterisation theorems for four variants of Sugeno integrals. These results pave the way to decision-theoretic axiomatizations of these natural qualitative aggregations.
\end{abstract}

Keywords: Sugeno integral, residuated implications, multifactorial evaluation

\section{Introduction}

In multi-criteria decision making, Sugeno integrals $[20,21]$ are commonly used as qualitative aggregation functions [16]. The definitions of these integrals are based on a monotonic set-function named capacity or fuzzy measure that aims to qualitatively represent the likelihood of sets of possible states of nature, the importance of sets of criteria, etc. These set functions are currently used in many areas such as uncertainty modeling $[8,9]$, multiple criteria aggregation [15] or in game theory [18].

Capacities can be exploited in different ways when aggregating local ratings of objects according to various criteria, and different qualitative integrals have been laid bare to that effect [10]. In the case of Sugeno integrals the capacity is used as a bound that restricts the global evaluation from below or from above. In the other cases, the capacity is considered as a tolerance threshold such that overcoming it is sufficient to reach the best global evaluation of the object under study. When this threshold is not reached there are two possibilities. Either the local rating remains as it stands or it is modified: improved if the criterion is little important, downgraded if it is important. These possibilities give rise to new aggregation operations in the qualitative setting, namely the soft integrals and the drastic integrals, respectively [10].

These qualitative integrals are valued in a scale equipped with a residuated implication and an involutive negation $[10,11]$. More precisely, the evaluation scale is both a totally ordered Heyting algebra and a Kleene algebra.

The aim of this paper is to establish characterization theorems for the soft qualitative integrals and the drastic qualitative integrals. These results are stated in Theorems 5.6, 5.8, 6.5, 6.7. Such characterization results pave the way to an axiomatization of such criteria in terms of preference relations between evaluated objects, as previously done for Sugeno integrals $[8,9]$.

The paper is organized as follows. Section 2 presents the framework and notations, Section 3 deals with the characteristic properties of soft integrals and Section 4 with those of drastic integrals.

\section{Framework and notations}

We adopt the terminology and notations usual in multi-criteria decision making, where some alternatives are evaluated according to a common set $C=\{1, \ldots, n\}=[n]$ of criteria. A common evaluation scale $L$ is assumed to provide ratings according to the criteria: each alternative is thus identified with a function $f \in L^{C}$ which maps every criterion $i$ of $C$ to the local rating $f_{i}$ of the alternative with regard to this criterion. We assume that $L$ is a totally ordered set with 1 and 0 as top and bottom, respectively ( $L$ may be the real unit interval $[0,1]$ for instance). We denote by $\wedge$ and $\vee$ the minimum and maximum operation on $L$. In addition, we assume that $L$ is equipped with a unary order reversing involutive operation $t \mapsto 1-t$. In the definitions of the qualitative integrals that we consider in the paper, the relative weights of the sets of criteria are modeled through a capacity, which is an order-preserving map $\gamma: 2^{C} \rightarrow L$ that satisfies $\gamma(\varnothing)=0$ and $\gamma(C)=1$. The conjugate capacity $\gamma^{c}$ of $\gamma$ is defined by $\gamma^{c}(A)=1-\gamma(\bar{A})$ for every $A \subseteq C$, where $\bar{A}$ denotes the complement of $A$.

In order to define the qualitative integrals considered in this paper, we also equip $L$ with binary operations denoted by $\rightarrow_{D}, \rightarrow_{G}, \rightarrow_{G C}, \star_{D}, \star_{G}, \star_{G C}$ 
and defined by

$$
\begin{gathered}
a \rightarrow_{D} b=(1-a) \vee b \\
a \rightarrow_{G} b=1 \quad \text { if } a \leq b, \quad \text { and } b \quad \text { otherwise, } \\
a \rightarrow_{G C} b=(1-b) \rightarrow_{G}(1-a) \\
a \star b=1-(a \rightarrow(1-b))
\end{gathered}
$$

for every $a, b \in L$ and every $(\rightarrow, \star) \in\left\{\left(\rightarrow_{D}, \star_{D}\right),\left(\rightarrow_{G}\right.\right.$, $\left.\left.\star_{G}\right),\left(\rightarrow_{G C}, \star_{G C}\right)\right\}$. Moreover, it can be checked that $a \rightarrow b=1-(a \star(1-b))$ for the three above pairs. Besides the other pairs $(\rightarrow, \star) \in\left\{\left(\rightarrow_{G}\right.\right.$ ,$\left.\left.\star_{D}\right),\left(\rightarrow_{D}, \star_{G}\right),\left(\rightarrow_{G C}, \star_{G C}\right)\right\}$ are linked by residuation, namely for these three latter pairs, we have $a \rightarrow b=\sup \{x \in L \mid a \star x \leq b\}$ [4]. Note that the operations $\rightarrow_{D}, \rightarrow_{G}$, and $\rightarrow_{G C}$ are the Kleene Dienes, Gödel and contraposed Gödel implications, respectively. It is convenient to note that by definition of $\star_{G}$ we have $a \star_{G} b=0$ if $a \leq 1-b$, and $a \star_{G} b=b$ otherwise, so it is a non-commutative conjunction. In particular,

$a \star_{G} 0=0, \quad b \star_{G} 1=1, \quad 0 \star_{G} a=0, \quad 1 \star_{G} a=a$,

for every $a \in L$ and $b \in L \backslash\{0\}$. Also, it is easily checked that

$$
a \star_{G C} b=b \star_{G} a,
$$

for every $a, b \in L$. Finally, note that $\star_{D}$ is just the minimum $\wedge$.

Now we can recall the definitions of the qualitative integrals that we consider in this paper [10].

Definition 2.1. Let $\star \in\left\{\star_{D}, \star_{G}, \star_{G C}\right\}$ and $\gamma$ be a capacity. We define the mapping $\int_{\gamma}^{\star}: L^{C} \rightarrow L$ by setting

$$
\int_{\gamma}^{\star} f=\bigvee_{A \subseteq C}\left(\gamma(A) \star \bigwedge_{i \in A} f_{i}\right),
$$

for all $f \in L^{C}$. For $\rightarrow \in\left\{\rightarrow_{D}, \rightarrow_{G}, \rightarrow_{G C}\right\}$, we define the mapping $\int_{\gamma}^{\rightarrow}: L^{C} \rightarrow L$ by setting

$$
\int_{\gamma}^{\rightarrow} f=\bigwedge_{A \subseteq C}\left(\gamma^{c}(A) \rightarrow \bigvee_{i \in A} f_{i}\right)
$$

for all $f \in L^{C}$.

The integrals associated to the Gödel (resp. contraposed Gödel) implication or to the conjunction $\star_{G}\left(\right.$ resp. $\left.\star_{G C}\right)$ related by (1) to the implication, are called soft integrals (resp. drastic integrals). These names need some comments. If $\rightarrow$ is Gödel implication, the term $\gamma^{c}(A) \rightarrow \bigvee_{i \in A} f_{i}$ is 1 if some $f_{i}$ is large enough in $A$, and is equal to the maximum value of $f$ on $\mathrm{A}$ otherwise. In particular, the vector with components $\gamma^{c}(\{i\}) \rightarrow f_{i}$ is a softened version of $f$. On the other hand, the term $\gamma^{c}(A) \rightarrow \bigvee_{i \in A} f_{i}$, when $\rightarrow$ is the contrapositive version of Gödel implication becomes 1 or $\gamma^{c}(A)$, so that this integral does not use the local ratings $f_{i}$ but for testing its position with respect to the weights, in such a way that the local criterion scales become binary ( 1 or the value of some weight). This is what we mean by a drastic behavior.

Also, for every capacity $\gamma$, we have $\int_{\gamma}^{\star_{D}}=\int_{\gamma}^{\rightarrow_{D}}=$ $\int_{\gamma}$ where $\int_{\gamma}$ is a mapping $L^{C} \rightarrow L$ called Sugeno integral, which is equivalently expressed by [17]:

$$
\int_{\gamma} f=\bigvee_{A \subseteq C}\left(\gamma(A) \wedge \bigwedge_{i \in A} f_{i}\right)=\bigwedge_{A \subseteq C}\left(\gamma(\bar{A}) \vee \bigvee_{i \in A} f_{i}\right)
$$

\section{Simple qualitative aggregation schemes on positive scales}

This section focuses on special cases of the above qualitative aggregation functions. They essentially generalize two elementary qualitative aggregation schemes:

- The first one, which maps $f \in L^{C}$ to $\bigwedge_{i=1}^{n} f_{i}$, is pessimistic and very demanding; namely, in order to obtain a good global evaluation, an object needs to satisfy all the criteria.

- The second one, which maps $f \in L^{C}$ to $\bigvee_{i=1}^{n} f_{i}$, is optimistic and very loose; namely, one fulfilled criterion is enough to obtain a good global evaluation.

These two aggregation schemes can be slightly generalised by means of importance levels or priorities $\pi_{i} \in L$, on the criteria $i \in[n]$. Suppose $\pi_{i}$ is increasing with the importance of $i$. A fully important criterion has importance weight $\pi_{i}=1$. In the following, we assume $\pi_{i}>0$ for every $i \in[n]$, i.e., there is no useless criterion. In this section, we also assume $\pi_{i}=1$, for some criterion $i$ (the most important one). These importance levels can alter each local evaluation $f_{i}$ in different manners. More precisely, $\pi_{i}$ can act as a saturation threshold that blocks the global score under or above a certain value dependent on the importance level of criterion $i$. Alternatively, $\pi_{i}$ can be considered as a threshold above which the decision-maker is perfectly satisfied and under which the local rating is altered or not. There are two such rating modification schemes. All of them use a pair (implication, conjunction) defined previously. Let us present all these cases in details.

\subsection{Saturation levels}

Here the importance weights act as saturation levels: they reduce the evaluation scale from above or from below. The rating $f_{i}$ is modified either into $\left(1-\pi_{i}\right) \vee f_{i} \in\left[1-\pi_{i}, 1\right]$, or into $\pi_{i} \wedge f_{i} \in\left[0, \pi_{i}\right]$. A fully important criterion can affect the whole global score scale.

- In a demanding aggregation, all the important criteria have to be satisfied, which justifies the prioritized minimum [5]:

$$
\operatorname{SLMIN}_{\pi}(f)=\bigwedge_{i=1}^{n}\left(\left(1-\pi_{i}\right) \vee f_{i}\right)=\bigwedge_{i=1}^{n} \pi_{i} \rightarrow_{D} f_{i}
$$


Hence an important criterion can alone bring the overall score very low and a criterion $i$ that is of little importance cannot downgrade the overall score under a certain level $1-\pi_{i}$. A fully important criterion $\left(\pi_{i}=1\right)$ acts as a veto as it can lead to a zero global score if violated. This is why under this aggregation scheme, such criteria can actually be viewed as soft constraints [3]. The weights $\pi_{i}$ are priorities, that affect the level of acceptance of objects that violate criteria.

- In a loose aggregation we just need to satisfy one important criterion, which justifies the prioritized maximum $[5,19]$ :

$$
S L M A X_{\pi}(f)=\bigvee_{i=1}^{n} \pi_{i} \wedge f_{i}=\bigvee_{i=1}^{n} \pi_{i} \star_{D} f_{i} .
$$

In this case an important criterion is one that alone can bring a good overall score (a maximal one for a fully important criterion) and a not important criterion can never alone bring the overall score higher than $\pi_{i}$.

It is well-known that if the evaluation scale of the local ratings $f_{i}$ is reduced to $\{0,1\}$ (Boolean criteria) then letting $A_{f}=\left\{i: f_{i}=1\right\}$ be the set of criteria satisfied by object $f, S L M A X_{\pi}(f)=\bigvee\left\{\pi_{i}\right.$ : $\left.i \in A_{f}\right\}=\Pi\left(A_{f}\right)$ is a possibility measure [22] (i.e, a capacity $\Pi$ that satisfies $\Pi(A \cup B)=\Pi(A) \vee \Pi(B)$ for every $A, B \subseteq C)$, and $S L M I N_{\pi}(f)=\bigwedge\left\{1-\pi_{i}\right.$ : $\left.i \notin A_{f}\right\}=N\left(A_{f}\right)$ is a necessity measure [6] (i.e., a capacity $N$ that satisfies $N(A \cap B)=N(A) \wedge N(B)$ for every $A, B \subseteq C)$. Note that we have the following De Morgan-like property, that extends the well-known duality $\Pi(A)=1-N(\bar{A})$, where $\bar{A}$ is the complement of $A$, to graded tuples $f$ :

$$
S L M A X_{\pi}(f)=1-\operatorname{SLMIN}_{\pi}(1-f) .
$$

\subsection{Softening thresholds}

The importance weight $\pi_{i}$ of a criterion can be considered as a threshold that if passed by the corresponding local rating, is sufficient to obtain a full local score. Namely, if $f_{i}$ is greater than $\pi_{i}$ then the local rating becomes maximal, i.e., equals 1 . Otherwise, if $f_{i}$ is less than $\pi_{i}$, then the local rating remains as it stands. Clearly, the effect of the weight $\pi_{i}$ on the original local rating $f_{i}$ is to turn it into $\pi_{i} \rightarrow_{G} f_{i}$.

- The demanding soft aggregation is obtained by replacing $\rightarrow_{D}$ by $\rightarrow_{G}$. We get

$$
\operatorname{STMIN}_{\pi}(f)=\bigwedge_{i=1}^{n} \pi_{i} \rightarrow_{G} f_{i} .
$$

The idea is still that the evaluated item should get good grades for all important criteria. In this case, a criterion is all the less important as the required rating for considering it fulfilled is low. A fully important criterion is considered satisfied only if $f_{i}=1$. A criterion $i$ with a low importance weight $\pi_{i}$ is fully satisfied even by objects for which $f_{i}$ is low, provided that this local rating is above $\pi_{i}$.

- We can define the corresponding loose aggregation, changing $\wedge$ into the conjunction $\star_{G}$ associated to $\rightarrow_{G}$ in the SLMAX aggregation scheme:

$$
S T M A X_{\pi}(f)=\bigvee_{i=1}^{n} \pi_{i} \star_{G} f_{i} .
$$

Since $\pi_{i} \star_{G} f_{i}=0$ as soon as $f_{i} \leq 1-\pi_{i}$, it means that for a criterion of low importance, the local rating for criterion $i$ must be very high (at least $1-\pi_{i}$ ) to influence the global score and it is eliminated otherwise. On the contrary, an important criterion $i$ may affect the global rating even if the corresponding local rating is low.

It is easy to see that

- $\operatorname{STMIN}_{\pi}(f)=1$ if and only if $f_{i} \geq \pi_{i}$ for every $i \in[n]$, that is if and only if the local ratings reach at least the levels prescribed by the importance thresholds. Note that is a rather unsurprising demand.

- $S T M I N_{\pi}(f)=0$ if and only if there is some $i \in$ $[n]$ such that $f_{i}=0$, that is, if some criterion is totally violated.

- $S T M A X_{\pi}(f)=1$ if and only if there is some $i \in[n]$ such that $f_{i}=1$, that is, if some criterion is totally satisfied.

- $S T M A X_{\pi}(f)=0$ if and only if $f_{i} \leq 1-\pi_{i}$ for every $i \in[n]$, that is, if no criterion passes the rating threshold $1-\pi_{i}$.

In fact, with $S T M I N_{\pi}(f)$, the weights select violated criteria that alone are enough to eliminate $f$, and with $S T M A X N_{\pi}(f)$, the weights select satisfied criteria that alone are enough to accept $f$.

We have again the following De Morgan-like duality:

$$
S T M A X_{\pi}(f)=1-S T M I N_{\pi}(1-f) .
$$

However, $S T M I N_{\pi}(f)$ and $S T M A X_{\pi}(f)$ cannot be considered as a proper generalization to fuzzy events of possibility and necessity measures, since when the $f_{i}$ 's belong to $\{0,1\}$ and $f$ is the characteristic function $\mu_{B}$ of set $B$, we do not get $\operatorname{STMIN}_{\pi}\left(\mu_{B}\right)=N(B)$, nor $\operatorname{STMAX} X_{\pi}\left(\mu_{B}\right)=$ $\Pi(B)$.

Indeed, in that case $S T M I N_{\pi}(f) \in\{0,1\}$ and $S T M A X_{\pi}(f) \in\{0,1\}$ as well. Namely $S T M I N_{\pi}\left(\mu_{B}\right)=1$ if $B=C$, 0 otherwise, and $S T M A X_{\pi}\left(\mu_{B}\right)=1$ if $B \neq \emptyset$, and 0 otherwise. In other words, everything happens as if weights were all equal to $1, S T M I N_{\pi}$ being a standard conjunction, and $S T M A X_{\pi}$ a standard disjunction. It is known [7] that the residuation-based extension of necessity measures to fuzzy events is not based on Gödel implication, but on its contrapositive form. 


\subsection{Drastic thresholdings}

Another way of handling importance weights is to downgrade or upgrade local ratings to a fixed value when they fail to reach the importance thresholds $\pi_{i}$, this prescribed value decreasing with the importance of the criterion. Namely, if $f_{i}<\pi_{i}$ then we set the rating to $1-\pi_{i}$. As a consequence, the modified rating is modeled by $\pi_{i} \rightarrow_{G C} f_{i}$, so that the local evaluation scale of criterion $i$ is reduced to pseudo-Boolean values in the set $\left\{1-\pi_{i}, 1\right\}$, which is a drastic way of handling graded ratings. Again we shall have demanding and loose aggregations.

- The demanding aggregation will be

$$
\operatorname{DTMIN}_{\pi}(f)=\bigwedge_{i=1}^{n} \pi_{i} \rightarrow_{G C} f_{i} .
$$

When violated (i.e. a threshold $\pi_{i}$ is missed), an important criterion alone may drastically downgrade the overall score, while the local rating according to an unimportant criterion may be upgraded (in each case, to $1-\pi_{i}$, which is low in the first case and high in the second case).

- The loose counterpart will be

$$
\operatorname{DTMAX} X_{\pi}(f)=\bigvee_{i=1}^{n} \pi_{i} \star_{G C} f_{i}
$$

An important criterion, if satisfied, can alone bring the overall score to a high value but an unimportant criterion, even if satisfied, cannot bring the overall score to a high value $\left(\pi_{i}\right.$ in each case).

Letting $A_{f}^{\downarrow}=\left\{i: f_{i}<\pi_{i}\right\}$, we observe that $\operatorname{DTMIN}_{\pi}(f)=N\left(A_{f}^{\downarrow}\right)$. Likewise denoting $A_{f}^{\uparrow}=$ $\left\{i: f_{i}>1-\pi_{i}\right\}$, we observe that $\operatorname{DTMAX}_{\pi}(f)=$ $\Pi\left(A_{f}^{\uparrow}\right)$, so that when $f_{i} \in\{0,1\}$, we do get necessity and possibility measures (and then, $A_{f}^{\downarrow}=\overline{A_{f}^{\uparrow}}$ ). We have again the following duality:

$$
\operatorname{DTMAX}_{\pi}(f)=1-\operatorname{DTMIN} N_{\pi}(1-f) .
$$

\subsection{Weighted min and max as special cases of qualitative integrals}

When in the proposed qualitative integrals subsumed by Definition 2.1 (Sugeno, soft, drastic) the capacity is restricted to a possibility or a necessity measure, we have the following result [12]:

Proposition 3.1. If $\gamma$ is a necessity measure $N$, then $\int_{N}^{\rightarrow G}=S T M I N_{\pi}$ and $\int_{N}^{\rightarrow G C}=D T M I N_{\pi}$.

If $\gamma$ is a possibility measure $\Pi$ then $\int_{\Pi}^{\star G}=$ $S T M A X_{\pi}$ and $\int_{\Pi}^{\star G C}=D T M A X_{\pi}$.

Note that we cannot exchange possibility and necessity measures in the above results, as the corresponding expressions no longer simplify.

\section{The case of Sugeno integrals: known facts}

A characterization theorem of the Sugeno integral is given in [1]. It can be stated in the following way. Recall that $f, g \in L^{C}$ are said to be comonotone, if for every $i, j \in[n]$, if $f(i)<f(j)$ then $g(i) \leq g(j)$, and if $g(i)<g(j)$ then $f(i) \leq f(j)$. It is equivalent to say that for every permutation $\sigma$ on $[n]$, we have $f(\sigma(1)) \leq \cdots \leq f(\sigma(n))$ if and only if $g(\sigma(1)) \leq$ $\cdots \leq g(\sigma(n))$. For every $A \subseteq C$, we denote by $\mathbf{1}_{A}$ the characteristic function $\mathbf{1}_{A}: C \rightarrow\{0,1\}$ of $A$. Besides, remember that $\wedge=\star_{D}$.

Theorem 4.1 ([1]). Let $I: L^{C} \rightarrow L$. There is a capacity $\gamma$ such that $I(f)=\int_{\gamma} f$ for every $f \in L^{C}$ if and only if the following properties are satisfied

1. $I(f \vee g)=I(f) \vee I(g)$, for any comonotone $f, g \in L^{C}$.

2. $I(a \wedge f)=a \star_{D} I(f)$, for every $a \in L$ and $f \in L^{C}$.

3. $I\left(\mathbf{1}_{C}\right)=1$

Equivalently, conditions (1-3) can be replaced by conditions (1'-3') below.

1. $I(f \wedge g)=I(f) \wedge I(g)$, for any comonotone $f, g \in L^{C}$.

2. $I\left(a \rightarrow_{D} f\right)=a \rightarrow_{D} I(f)$, for every $a \in L$ and $f \in L^{C}$.

3. $I(0)=0$

In the next sections, we aim to obtain characterization results of the drastic and soft integrals in the spirit of Theorem 4.1. Informally speaking, the method we use to search for an axiomatization of $\int_{\gamma}^{\star}$ where $\star \in\left\{\star_{G}, \star_{G C}\right\}$ is the following. We try to split $f$ in small chunks in such a way that $\int_{\gamma}^{\star}$ commutes with the decomposition and such that the values of $\int_{\gamma}^{\star}$ on any of these chunks is easily mastered.

We end this section by giving the following alternative formulations of these integrals.

Remark 4.2. For any $f \in L^{C}$, any inequality $\bowtie \in$ $\{\leq, \geq,<,>\}$, and any $a \in L$ we denote by $\{f \bowtie a\}$ the set $\left\{i \in C \mid f_{i} \bowtie a\right\}$. Moreover, we use the symbol ${ }_{(i)}$ to indicate that the indices have been rearranged so that $f_{(1)} \leq \cdots \leq f_{(n)}$, and $A_{(i)}$ stands for $\{(i), \ldots,(n)\}$ for every $i \in[n]$, with the convention $A_{(n+1)}=\varnothing$.

Proposition 4.3 ([12]). For any $\star \in\left\{\star_{G}, \star_{G C}\right\}$, any $\rightarrow \in\left\{\rightarrow_{G}, \rightarrow_{G C}\right\}$, any capacity $\gamma$ and any $f \in$ $L^{C} \rightarrow L$ we have

$$
\begin{gathered}
\int_{\gamma}^{\star} f=\bigvee_{i=1}^{n} \gamma\left(A_{(i)}\right) \star f_{(i)}, \\
\int_{\gamma}^{\rightarrow} f=\bigwedge_{i=1}^{n} \gamma^{c}\left(\overline{A_{(i+1)}}\right) \rightarrow f_{(i)}, \\
\int_{\gamma}^{\star} f=\bigvee_{a \in L} \gamma(\{f \geq a\}) \star a,
\end{gathered}
$$




$$
\int_{\gamma}^{\rightarrow} f=\bigwedge_{a \in L} \gamma^{c}(\{f \leq a\}) \rightarrow a .
$$

Moreover, using the relation between the implication and the conjunction, it is not difficult to prove the following relation.

Lemma 4.4. If $(\star, \rightarrow) \in\left\{\left(\star_{G}, \rightarrow_{G}\right),\left(\star_{G C}, \rightarrow_{G C}\right)\right\}$, if $\gamma$ is a capacity and if $f \in L^{C}$, then we have $\int_{\gamma}^{\rightarrow} f=1-\int_{\gamma^{c}}^{\star}(1-f)$.

\section{Axiomatization of the soft integrals}

We begin this section with some technical results that will be used in the proofs of the characterization theorems of the soft and drastic integrals.

Lemma 5.1. If $f \in L^{C}$ then $f=\bigvee_{i=1}^{n} \mathbf{1}_{A_{(i)}} \star_{G} f_{(i)}$.

Proof: For any $i, k \in[n]$, we have $f_{(i)} \leq f_{k}$ if $k \in A_{(i)}$. It follows that $\mathbf{1}_{A_{(i)}}(k) \star_{G} f_{(i)}=0$ if $f_{k}<f_{(i)}$. We then have

$$
\begin{aligned}
\bigvee_{i=1}^{n} \mathbf{1}_{A_{(i)}}(k) \star_{G} f_{(i)} & =\bigvee\left\{f_{(i)} \mid f_{(i)} \leq f_{k}\right\} \\
& =f_{k}
\end{aligned}
$$

which is the desired result.

Lemma 5.2. For every capacity $\gamma$, the map $\int_{\gamma}^{\star_{G}}: L^{C} \rightarrow L$ is order-preserving.

Proof: The proof directly follows from the definition of the integral using the fact that the map $x \mapsto a \star_{G} x$ in order-preserving for every $a \in L$.

In order to prove the following Lemma, let us recall that for any two comonotone $f, g \in L^{C}$ and any $a \in L$ we have either $\{f \geq a\} \subseteq\{g \geq a\}$ or $\{g \geq a\} \subseteq\{f \geq a\}$.

Lemma 5.3. For any comonotone $f, g \in L^{C}$, we have $\int_{\gamma}^{\star_{G}}(f \vee g)=\int_{\gamma}^{\star_{G}} f \vee \int_{\gamma}^{\star_{G}} g$.

Proof: The inequality $\int_{\gamma}^{\star_{G}}(f \vee g) \geq \int_{\gamma}^{\star_{G}} f \vee$ $\int_{\gamma}^{\star_{G}} g$ follows from Lemma 5.2. Let us prove the other inequality. Let $a \in L$. If $\{f \geq a\} \subseteq\{g \geq a\}$ then $\{f \vee g \geq a\}=\{f \geq a\} \cup\{g \geq a\}=\{g \geq a\}$. It follows that

$$
\begin{aligned}
& \gamma(\{f \vee g \geq a\}) \star_{G} a=\gamma(\{g \geq a\}) \star_{G} a \\
\leq & \left(\gamma(\{g \geq a\}) \star_{G} a\right) \vee\left(\gamma(\{f \geq a\}) \star_{G} a\right) .
\end{aligned}
$$

By symmetry, (3) also holds whenever $\{g \geq a\} \subseteq$ $\{f \geq a\}$, i.e., inequality (3) holds for every $a \in L$. By Proposition 4.3 we then obtain

$$
\begin{aligned}
\int_{\gamma}^{\star_{G}}(f \vee g) \leq & \bigvee_{a \in L}\left(\left(\gamma(\{g \geq a\}) \star_{G} a\right)\right. \\
& \left.\vee\left(\gamma(\{f \geq a\}) \star_{G} a\right)\right) \\
& =\int_{\gamma}^{\star_{G}} f \vee \int_{\gamma}^{\star_{G}} g
\end{aligned}
$$

which gives the desired result.

Lemma 5.4. For a given $f$, assume the criteria are well-ordered such that $f_{(1)} \leq \cdots \leq f_{(n)}$; then for every $f \in L^{C}$ and every $\ell \in\{1, \ldots, n-1\}$,

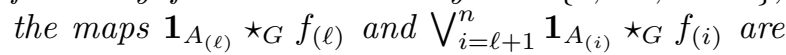
comonotone.

Proof: We represent both maps as vectors of components ordered according to $(1), \ldots,(n)$, so that $A_{(\ell)}=\{(\ell), \ldots,(n)\}$. In consequence:

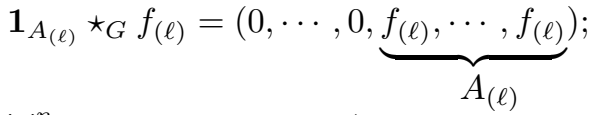

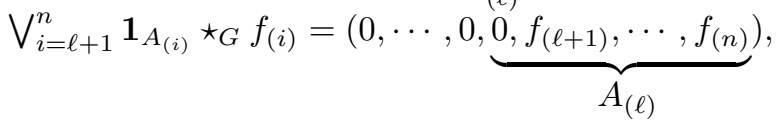

hence it is easy to check that the two maps are comonotone.

Lemma 5.5. For any capacity $\gamma$, any $B \subseteq C$ and any $a \in L$ we have $\int_{\gamma}^{\star_{G}}\left(\mathbf{1}_{B} \star_{G} a\right)=\gamma(B) \star_{G} a$. In particular, $\int_{\gamma}^{\star_{G}} \mathbf{1}_{B} \in\{0,1\}$ for every $B \subseteq C$, and $\int_{\gamma}^{\star_{G}} \mathbf{1}_{C}=1$.

Proof: We use Proposition 4.3 and evaluate the integral as $\int_{\gamma}^{\star_{G}} f=\bigvee_{i=1}^{n} \gamma\left(A_{(i)}\right) \star_{G} f_{(i)}$, where $f=\mathbf{1}_{B} \star_{G} a$. Note that if $i \in B$, then $\mathbf{1}_{B}(i) \star_{G} a=$ $a$ and 0 otherwise. So, there is $j$ such that $B=$ $A_{(j)}=\{(j), \ldots,(n)\}$. So we get $\int_{\gamma}^{\star_{G}}\left(\mathbf{1}_{B} \star_{G} a\right)=$ $\bigvee_{i \geq j}^{n} \gamma\left(A_{(i)}\right) \star_{G} a$, and the maximum is attained for $i=j$. Finally if $a=1$ note that $\mathbf{1}_{B}(i) \star_{G} a \in\{0,1\}$, and $\mathbf{1}_{C}(i) \star_{G} 1=1$.

We can now prove our first characterization result.

Theorem 5.6. Let $I: L^{C} \rightarrow L$ be a mapping. There is a capacity $\gamma$ such that $I(f)=\int_{\gamma}^{\star_{G}} f$ for every $f \in L^{C}$ if and only if the following properties are satisfied.

1. $I(f \vee g)=I(f) \vee I(g)$, for any comonotone $f, g \in L^{C}$.

2. There is a capacity $\lambda: 2^{C} \rightarrow L$ such that $I\left(\mathbf{1}_{A} \star_{G} a\right)=\lambda(A) \star_{G}$ a for every $a \in L$ and every $A \subseteq C$.

In that case, we have $\gamma=\lambda$.

Proof: Necessity is obtained by Lemmas 5.3 and 5.5. For sufficiency, assume that $I$ is a mapping that satisfies conditions 1 and 2 and let $f \in L^{C}$. By Lemma 5.1 we have $I(f)=I\left(\bigvee_{i=1}^{n} \mathbf{1}_{A_{(i)}} \star_{G} f_{(i)}\right)$. By assumption 1 and Lemma 5.4, the latter expression is in turn equal to $\bigvee_{i=1}^{n} I\left(\mathbf{1}_{A_{(i)}} \star_{G} f_{(i)}\right)$, and to $\bigvee_{i=1}^{n} \lambda\left(A_{(i)}\right) \star_{G} f_{(i)}$ by assumption 2 . Since $\lambda$ is a capacity, we conclude that $I(f)=\int_{\lambda}^{\star_{G}} f$ by Proposition 4.3.

Note that here, condition 3 in Th. 4.1, namely $I\left(\mathbf{1}_{C}\right)=1$, holds for free in Th. 5.6, since $\lambda$ is 
a capacity, while conditions 1 are identical in both theorems. Moreover, $\star_{G}$ in Th. 5.6 replaces $\star_{D}=\wedge$ in Th. 4.1. We use Theorem 5.6 and the two following lemmas to obtain a characterization of $\int_{\gamma}^{\rightarrow G}$.

Lemma 5.7. For any capacity $\gamma$, any $A \subseteq C$ and any $a \in L, \int_{\gamma}^{\rightarrow_{G}}\left(\mathbf{1}_{A} \rightarrow_{G} a\right)=\gamma^{c}(A) \rightarrow_{G}$ a. In particular, $\int_{\gamma}^{\rightarrow_{G}} \mathbf{1}_{C}=1$.

Proof: We first obtain

$$
\begin{aligned}
\int_{\gamma}^{\rightarrow_{G}}\left(\mathbf{1}_{A} \rightarrow_{G} a\right) & =1-\int_{\gamma^{c}}^{\star_{G}}\left(1-\left(\mathbf{1}_{A} \rightarrow_{G} a\right)\right) \\
& =1-\int_{\gamma^{c}}^{\star_{G}}\left(\mathbf{1}_{A} \star_{G}(1-a)\right),
\end{aligned}
$$

where the first identity is obtained by Lemma 4.4 and the second by Equation (1). By Lemma 5.5, the latter expression is in turn equal to $1-\left(\gamma^{c}(A) \star_{G}(1-a)\right)$, and to $\gamma^{c}(A) \rightarrow_{G}$ a using again identity (1).

Theorem 5.8. Let $I: L^{C} \rightarrow L$ be a mapping. There is a capacity $\gamma$ such that $I(f)=\int_{\gamma}^{{ }_{G}} f$ for every $f \in L^{C}$ if and only if the following properties are satisfied.

1. $I(f \wedge g)=I(f) \wedge I(g)$, for any comonotone $f, g \in L^{C}$.

2. There is a capacity $\rho: 2^{C} \rightarrow L$ such that $I\left(\mathbf{1}_{A} \rightarrow_{G} a\right)=\rho(A) \rightarrow_{G}$ a for every $a \in L$.

If these conditions are satisfied then $\gamma=\rho^{c}$.

Proof: Necessity follows easily by Lemma 5.7 and by combining Lemma 4.4 with Lemma 5.3. For sufficiency, let $I$ be a mapping satisfying conditions 1 and 2 of the statement and define $I^{\prime}: L^{C} \rightarrow L$ by setting $I^{\prime}(f)=1-I(1-f)$ for every $f \in L^{C}$. We obtain from our assumptions that $I^{\prime}$ satisfies assumptions 1 and 2 of Theorem 5.6 for $\lambda=\rho$. It follows that $I^{\prime}(f)=\int_{\rho}^{\star_{G}} f$, whence $I(f)=\int_{\rho^{c}}^{\vec{\rightarrow}_{G}} f$ for every $f \in L^{C}$ by Lemma 4.4.

\section{Axiomatization of the drastic integrals}

We apply a similar method to find a characterization of the soft integrals. First note that from Lemma 5.1 we immediately obtain by definition of $\star_{G C}$ that

$$
f=\bigvee_{i=1}^{n} f_{(i)} \star_{G C} \mathbf{1}_{A_{(i)}},
$$

for every $f \in L^{C}$. With this assumption, we also deduce from Lemma 5.4 that if $\ell \in\{1, \ldots, n-1\}$, then the maps $f_{(\ell)} \star_{G C} \mathbf{1}_{A_{(\ell)}}$ and $\bigvee_{i=\ell+1}^{n} f_{(i)}{ }_{G C} \mathbf{1}_{A_{(i)}}$ are comonotone. Finally, since the map $x \mapsto a \star_{G C} x$ is order-preserving for every $a \in L$, we directly deduce the following lemma.

Lemma 6.1. For every capacity $\gamma$, the map $\int_{\gamma}^{\star_{G C}}: L^{C} \rightarrow L$ is order-preserving.
Moreover, an analysis of the proof of Lemma 5.3 shows that we can substitute $\star_{G}$ by $\star_{G C}$ in this proof, i.e. we have the following lemma.

Lemma 6.2. For every capacity $\gamma$ and every comonotone $f, g \in L^{C}$, we have $\int_{\gamma}^{\star_{G C}}(f \vee g)=$ $\int_{\gamma}^{\star_{G C}} f \vee \int_{\gamma}^{\star_{G C}} g$.

Lemma 6.3. For every capacity $\gamma$, every $A \subseteq C$ and every $a \in L, \int_{\gamma}^{\star_{G C}}\left(a \star_{G C} \mathbf{1}_{A}\right)=a \star_{G} \gamma(A)$. In particular, $\int_{\gamma}^{\star G C} \mathbf{1}_{C}=1$.

Proof: We use the expression from Prop. 4.3 and evaluate the integral $\int_{\gamma}^{\star_{G C}}\left(a \star_{G C}\right.$ $\left.\mathbf{1}_{A}\right)=\bigvee_{B \subset C} \gamma(B) \star_{G C} \wedge_{i \in B}\left(a \star_{G C} \mathbf{1}_{A}(i)\right)=$ $\bigvee_{B \subseteq C} \wedge_{i \in B}\left(\mathbf{1}_{A}(i) \star_{G} \quad a\right) \star_{G} \gamma(B)$. However $\wedge_{i \in B}\left(\mathbf{1}_{A}(i) \star_{G} a\right)=a$ if $B \subseteq A$ and 0 otherwise. So $\int_{\gamma}^{\star_{G C}} f=\bigvee_{B \subseteq A} a \star_{G} \gamma(B)$, attained for $B=A$. It is clear that if $A=C$ and $a=1$ the result is 1 .

In particular, we have $\int_{\gamma}^{\star_{G C}} \mathbf{1}_{A}=\gamma(A)$ for every $A \subseteq C$, which entails the following property.

Lemma 6.4. For any capacity $\gamma$, any $A \subseteq C$ and $a \in L$, we have $\int_{\gamma}^{\star_{G C}} a \star_{G C} \mathbf{1}_{A}=a \star_{G} \int_{\gamma}^{\star_{G C}} \mathbf{1}_{A}$.

We are ready to prove the following characterization result.

Theorem 6.5. Let $I: L^{C} \rightarrow L$ be a mapping. There is a capacity $\gamma$ such that $I(f)=\int_{\gamma}^{\star G C} f$ for every $f \in L^{C}$ if and only if the following properties are satisfied.

1. $I(f \vee g)=I(f) \vee I(g)$, for any comonotone $f, g \in L^{C}$.

2. For every $A \subseteq C$ and every $a \in L$ we have $I\left(\mathbf{1}_{A} \star_{G} a\right)=I\left(\mathbf{1}_{A}\right) \star_{G C} a$.

3. $I\left(\mathbf{1}_{C}\right)=1$.

If these conditions are satisfied then $\gamma$ is defined by $\gamma(A)=I\left(\mathbf{1}_{A}\right)$ for every $A \subseteq C$.

Proof: Necessity is obtained by Lemmas $6.1-$ 6.4. For sufficiency, assume that $I: L^{C} \rightarrow L$ is a mapping that satisfies assumptions $3-2$ and let $f \in L^{C}$. By identity (4) we have $I(f)=$ $I\left(\bigvee_{i=1}^{n} \mathbf{1}_{A_{(i)}} \star_{G} f_{(i)}\right)$. By assumption 1, the latter expression is in turn equal to $\bigvee_{i=1}^{n} I\left(\mathbf{1}_{A_{(i)}} \star_{G} f_{(i)}\right)$, and to $\bigvee_{i=1}^{n} I\left(\mathbf{1}_{A_{(i)}}\right) \star_{G C} f_{(i)}$ by assumption 2 .

Let $\lambda: 2^{C} \rightarrow L$ be the set function defined by $\lambda(A)=I\left(\mathbf{1}_{A}\right)$ for every $A \subseteq C$. We prove that $\lambda$ is a capacity. From assumption 3 , we obtain successively $\lambda(C)=I\left(\mathbf{1}_{C}\right)=1$. Similarly, we obtain from assumptions 3 and 2 :

$$
\lambda(\emptyset)=I(0)=I\left(0 \star_{G C} \mathbf{1}_{C}\right)=0 \star_{G} \lambda(C)=0,
$$

which gives $\lambda(\varnothing)=0$. Finally, for every $A \subseteq B$ we have $1 \star_{G C} \mathbf{1}_{A} \leq 1 \star_{G C} \mathbf{1}_{B}$, which gives by conditions 1 and 2

$$
\begin{aligned}
\lambda(A) & =\lambda(A) \star_{G} 1 \\
& =I\left(1 \star_{G C} \mathbf{1}_{A}\right) \leq I\left(1 \star_{G C} \mathbf{1}_{B}\right) \\
& =1 \star_{G} \lambda(B)=\lambda(B)
\end{aligned}
$$


and we conclude that $\lambda$ is a capacity.

Finally, we obtain $I(f)=\int_{\lambda}^{\star G C} f$ by Lemma 4.3.

Clearly, this result parallels previous Theorems 4.1 and 5.6, with in particular identical conditions 1. Moreover, the difference between soft and drastic (conjunction-based) integrals is characterized by two expressions of condition 2 with the same lefthand part, where $\star_{G C}$ replaces $\star_{G}$ in the right-hand part (which should not come as a surprise).

We proceed further to obtain a characterization of the class of integrals $\int_{\gamma}^{\rightarrow_{G C}}$.

Lemma 6.6. For any capacity $\gamma$, any $A \subseteq C$ and $a \in L$, we have $\int_{\gamma}^{\rightarrow_{G C}}\left(a \rightarrow_{G C} \mathbf{1}_{A}\right)=a \rightarrow_{G} \gamma(A)$. In particular, $\int_{\gamma}^{\rightarrow_{G C}} \mathbf{1}_{A}=\gamma(A)$ and $\int_{\gamma}^{\rightarrow_{G C}} \mathbf{1}_{C}=1$.

Proof: We first obtain

$$
\begin{aligned}
\int_{\gamma}^{\rightarrow_{G C}}\left(a \rightarrow_{G C} \mathbf{1}_{A}\right) & =1-\int_{\gamma^{c}}^{\star_{G C}}\left(a \rightarrow_{G C} \mathbf{1}_{A}\right) \\
& =1-\int_{\gamma^{c}}^{\star_{G C}}\left(a \star_{G C} \mathbf{1}_{\bar{A}}\right),
\end{aligned}
$$

where the first identity is obtained by Lemma 4.4 . By Lemma 6.3, the latter expression is in turn equal to $1-\left(a \star_{G} \gamma^{c}(\bar{A})\right)$, and finally to $a \rightarrow_{G} \gamma(A)$. The particular cases are obtained by noting that $\mathbf{1}_{A}=1 \rightarrow_{G C} \mathbf{1}_{A}$ for every $A \subseteq C$.

Theorem 6.7. Let $I: L^{C} \rightarrow L$ be a mapping. There is a capacity $\gamma$ such that $I(f)=\int_{\gamma}^{\rightarrow_{G C}} f$ for every $f \in L^{C}$ if and only if the following properties are satisfied.

$$
\begin{aligned}
& \text { 1. } I(f \wedge g)=I(f) \wedge I(g) \text {, for any comonotone } \\
& f, g \in L^{C} \text {. } \\
& \text { 2. For every } A \subseteq C \text { and } a \in L \text { we have } I\left(a \rightarrow_{G C}\right. \\
& \left.\mathbf{1}_{A}\right)=a \rightarrow_{G} I\left(\mathbf{1}_{A}\right) \text {. } \\
& \text { 3. } I(0)=0 .
\end{aligned}
$$

If these conditions are satisfied then $\gamma$ is defined by $\gamma(A)=I\left(\mathbf{1}_{A}\right)$ for every $A \subseteq C$.

Proof: Necessity follows easily by Lemma 6.6 and by combining Lemma 4.4 with Lemmas 6.1 and 6.2. For sufficiency, let $I$ be a mapping satisfying conditions $3-2$ of the statement and define $I^{\prime}$ : $L^{C} \rightarrow L$ by setting $I^{\prime}(f)=1-I(1-f)$ for every $f \in L^{C}$. We obtain from our assumptions that $I^{\prime}$ satisfies assumptions 3 and 1 of Th. 6.5. Let us prove that $I^{\prime}$ also satisfies condition 2 of Th. 6.5.

We first obtain by definition that

$$
\begin{aligned}
I^{\prime}\left(a \star_{G C} \mathbf{1}_{A}\right) & =1-I\left(1-\left(a \star_{G C} \mathbf{1}_{A}\right)\right) \\
& =1-I\left(a \rightarrow_{G C} \mathbf{1}_{\bar{A}}\right) .
\end{aligned}
$$

The latter expression is equal to $1-\left(a \rightarrow_{G} I\left(\mathbf{1}_{\bar{A}}\right)\right)$ by assumption 2 and finally to $a \star_{G}\left(1-I\left(\mathbf{1}_{\bar{A}}\right)\right)=$ $a \star_{G} I^{\prime}\left(\mathbf{1}_{A}\right)$. It follows that $I^{\prime}(f)=\int_{\gamma}^{\star_{G C}} f$ where $\gamma$ is defined by $\gamma(A)=I^{\prime}\left(\mathbf{1}_{A}\right)$ for every $A \subseteq C$. Then we obtain by definition of $I^{\prime}$ that

$$
I(f)=1-\int_{\gamma}^{\star_{G C}}(1-f)=\int_{\gamma^{c}}^{\rightarrow_{G C}} f .
$$

Moreover $\gamma^{c}(A)=1-\gamma(\bar{A})=1-I^{\prime}\left(1-\mathbf{1}_{A}\right)=I\left(\mathbf{1}_{A}\right)$ for every $A \subseteq C$. We conclude that $I(f)=\int_{\rho}^{\rightarrow G C} f$ where $\rho(A)=I\left(\mathbf{1}_{A}\right)$ for every $A \subseteq C$.

Again there is a striking parallel between Th. 4.1, 5.8 and 6.7. In Th. 5.8, as $\gamma$ is a capacity, we get $I(0)=0$.

\section{Future works}

Based on the above characterisation results, there are two points that need further investigations. First, it looks natural to specialize the main theorems $5.6,5.8,6.5,6.7$ to the various weighted versions of min and max, whereby the capacity is restricted to possibility and necessity measures. The natural idea is to drop the assumption of comonotonicity of $f$ and $g$ in those theorems. This is especially clear for drastic integrals in the case of $\mathrm{Th}$. 6.5 where $\gamma(A)=I\left(\mathbf{1}_{A}\right)$. Then, the maxitivity of $I$ implies the one of $\gamma$, and thus $I=D T M A X$. Likewise, for Th. 6.7, for which the minitivity of $I$ implies the one of $\gamma$, and thus $I=D T M I N$. It is less obvious for the soft integrals, that do not straightforwardly generalize possibility and necessity measures.

Another natural question is to carry the representation results over to preference relations between alternatives $f, g$, looking for properties of these preference relations which ensure they can be represented by soft or drastic integrals, generalizing the already existing results for Sugeno integrals [8].

Yet another problem is to find characteristic properties of residuation integrals based on Łukasiewicz implication [13]. More generally, finding characteristic properties of integrals similar to those in Def. 2.1 based on other pairs $(\rightarrow, \star)$ where $\star \neq \wedge$ is an interesting question. Indeed, structures similar to $\left(\rightarrow_{D}, \rightarrow_{G}, \rightarrow_{G C}, \star_{D}, \star_{G}, \star_{G C}\right)$ (induced by the minimum, as recalled in the $2 \mathrm{~d}$ paragraph of Section 2 ), can be found. In particular, we may think of

i the pair $\left(a \rightarrow b=\left\{\begin{array}{l}1 \text { if } a \leq b, \\ a \rightarrow_{D} b \text { if } a>b,\end{array}\right.\right.$ and $\left.a \star b=\min \left(a \star_{G} b, a \star_{G C} b\right)\right)$, where $\star$ is the nilpotent minimum;

ii general pairs $(\rightarrow, \star)$ where $\star$ is a left-continuous triangular norm, and $a \rightarrow b=1-(a \star(1-b))$, since the above structure based on 3 conjunctions and 3 implications still holds [14], even if reduced to a single pair for nilpotent t-norms.

iii the qualitative pairs $(\rightarrow, \star)$ associated with the 6-tuple of implications and conjunctions $\left(\rightarrow_{R G}\right.$, $\left.\rightarrow_{X}, \rightarrow_{X C}, \star_{M}, \star_{l T}, \star_{r T C}\right)$, defined by 


$$
\begin{aligned}
& \text { a } a \rightarrow_{R G} b=\left\{\begin{array}{l}
1 \text { if } a \leq b, \\
0 \text { if } a>b ;
\end{array}\right. \\
& \mathbf{b} a \rightarrow_{X} b=\left\{\begin{array}{l}
1 \text { if } b=1, \\
1-a \text { if } b \neq 1 ;
\end{array}\right. \\
& \text { c } a \rightarrow_{X C} b=\left\{\begin{array}{l}
1 \text { if } a=0, \\
b \text { if } a \neq 0 ;
\end{array}\right. \\
& \text { d } a \star_{M} b=\left\{\begin{array}{l}
1 \text { if } a>1-b, \\
0 \text { if } a \leq 1-b ;
\end{array}\right. \\
& \text { e } a \star_{l T} b=\left\{\begin{array}{l}
0 \text { if } b=0, \\
a \text { if } b \neq 0 ;
\end{array}\right. \\
& \text { f } a \star_{r T C} b=\left\{\begin{array}{l}
0 \text { if } a=0, \\
b \text { if } a \neq 0 .
\end{array}\right.
\end{aligned}
$$

This largely ignored 6-tuple (except in the three-valued case, see [2]), uses Rescher-Gaines implication $\rightarrow_{R G}$, generated by the symmetrical conjunction $\star_{M}$ (which expresses that $(a, b)$ is fully acceptable if their mean is strictly greater than $1 / 2$, and fully rejected otherwise). As can be checked, it satisfies all the properties described in the second paragraph of Section 2, namely for every $a, b \in L$, we have:

(i) for all $(\rightarrow, \star) \in\left\{\left(\rightarrow_{R G}, \star_{M}\right),\left(\rightarrow_{X}, \star_{l T}\right)\right.$, $\left.\left(\rightarrow_{X C}, \star_{r T C}\right)\right\}, a \star b=1-(a \rightarrow(1-b))$ and $a \rightarrow b=1-(a \star(1-b))$,

(ii) the pairs $(\rightarrow, \star) \in\left\{\left(\rightarrow_{X}, \star_{M}\right),\left(\rightarrow_{R G}, \star_{l T}\right)\right.$, $\left.\left(\rightarrow_{X C}, \star_{r T C}\right)\right\}$ are linked by residuation,

(iii) $a \rightarrow_{X C} b=(1-b) \rightarrow_{X}(1-a)$ and $\beta \star_{l T} a=a \star_{r T C} b$.

The similarity between the 6-tuples $\left(\rightarrow_{D}, \rightarrow_{G}\right.$, $\left.\rightarrow_{G C}, \star_{D}, \star_{G}, \star_{G C}\right)$ and $\left(\rightarrow_{R G}, \rightarrow_{X}, \rightarrow_{X C}, \star_{M}, \star_{l T}\right.$, $\left.\star_{r T C}\right)$ is striking. Especially, they both make sense in a purely qualitative setting.

\section{References}

[1] A. Chateauneuf, M. Grabisch, A. Rico. Modeling attitudes toward uncertainty through the use of the Sugeno integral. J. of Mathematical Economics, 44, 1084-1099, 2008.

[2] D. Ciucci, D. Dubois. A map of dependencies among three-valued logics. Inf. Sci. 250, 162-177, 2013.

[3] D. Dubois, H. Fargier, H. Prade. Possibility theory in constraint satisfaction problems: Handling priority, preference and uncertainty. Applied Intelligence, 6, 287-309, 1996.

[4] D. Dubois, H. Prade. A theorem on implication functions defined from triangular norms. Stochastica, VIII (3), 267-279, 1984.

[5] D. Dubois, H. Prade. Weighted minimum and maximum operations. Inf. Sci., 39, 205-210, 1986

[6] D. Dubois, H. Prade. Possibility Theory. Plenum, 1988.
[7] D. Dubois, H. Prade. Fuzzy rules in knowledgebased systems : Modelling gradedness, uncertainty and preference. In: An Introduction to Fuzzy Logic Applications in Intelligent Systems (R. Yager, L. Zadeh, eds.), Kluwer, 45-68, 1992.

[8] D. Dubois, H. Prade, R. Sabbadin. Qualitative decision theory with Sugeno integrals, In: M. Grabisch et al., eds., Fuzzy Measures and Integrals - Theory and Applications, Heidelberg, Physica Vrlg., 314-322, 2000.

[9] D. Dubois, H. Prade. R. Sabbadin. Decisiontheoretic foundations of qualitative possibility theory. Eur. J. Oper. Res., 128, 459-478, 2001.

[10] D. Dubois, H. Prade, A. Rico. Qualitative integrals and desintegrals: How to handle positive and negative scales in evaluation. In: Advances in Computational Intelligence, Part III, (IPMU'12), (S. Greco et al., eds.), Springer, CCIS 299, 306-316, 2012

[11] D. Dubois, H. Prade, A. Rico. Qualitative integrals and desintegrals - Towards a logical view. Proc. 9th Int. Conf. on Modeling Decisions for Artificial Intelligence (MDAI'12), LNCS 7647, Springer, 127-138, 2012.

[12] D. Dubois, H. Prade, A. Rico. Qualitative integrals and desintegrals as lower and upper possibilistic expectations. Proc. 8th Europ. Soc. for Fuzzy Logic and Technology Conf. (EUSFLAT'13), (J. Montero et al., eds.), Atlantis Press, 318-325, 2013

[13] A. Dvořák, M. Holčapek. Fuzzy integrals over complete residuated lattices. Proc. Joint ISFAEUSFLAT Conf. (Carvalho, J. P. et al., eds.), 357-362, 2009.

[14] J. Fodor. On fuzzy implication operators. Fuzzy Sets Syst., 42(3), 293-300, 1991.

[15] M. Grabisch. The application of fuzzy integrals in multicriteria decision making. Europ. J. of Operat. Res., 89 (3), 445-456, 1996.

[16] M. Grabisch, Ch. Labreuche. A decade of application of the Choquet and Sugeno integrals in multi-criteria decision aid. Annals of Oper. Res. 175, 247-286, 2010.

[17] J.-L. Marichal. On Sugeno integrals as an aggregation function. Fuzzy Sets Syst., 114, 347$365,2000$.

[18] D. Schmeidler, Cores of exact games. J. of Math. Anal. and Appl., 40 (1), 214-225, 1972.

[19] R. Yager, Possibilistic decision making. IEEE Trans. Syst., Man \& Cyber., 9, 388-392, 1979.

[20] M. Sugeno. Theory of Fuzzy Integrals and its Applications. PhD Thesis, Tokyo Institute of Technology, 1974.

[21] M. Sugeno. Fuzzy measures and fuzzy integrals: A survey. In: Fuzzy Automata and Decision Processes, (M. M. Gupta, et al., eds.), North-Holland, 89-102, 1977.

[22] L. A. Zadeh. Fuzzy sets as a basis for a theory of possibility. Fuzzy Sets \& Syst., 1, 3-28, 1978. 